\title{
X-ray emission from classical and recurrent novae observed with ROSAT
}

\author{
M. Orio ${ }^{1,2}$, J. Covington ${ }^{3}$, and H. Ögelman ${ }^{3}$ \\ 1 Osservatorio Astronomico di Torino, Strada Osservatorio, 20, 10025 Pino Torinese (TO), Italy \\ 2 Department of Astronomy, 475 N. Charter Str., Madison WI 53706, USA \\ 3 Physics Department, 1150 University Avenue, Madison WI 53706, USA
}

Received 7 February 2000 / Accepted 10 April 2001

\begin{abstract}
We have analysed 350 pointed and serendipitous observations of 108 different classical and recurrent novae in outburst and in quiescence, contained in the ROSAT archive. One aim was to search for super-soft X-ray sources and we found only 3 of them among post-novae. Thus, the super-soft X-ray phase of novae is relatively short lived (up to 10 years) and is observed only for up to $20 \%$ of novae. Most classical and recurrent novae instead emit hard X-rays (in the ROSAT band) in the first months after the outburst, with peak X-ray luminosity of a few times $10^{33} \mathrm{erg} \mathrm{s}^{-1}$. The emission, which we attribute to shocks in the nova ejecta, lasts at least $\simeq 2$ years and even much longer under special circumstances (like preexisting circumstellar material, or a prolonged wind phase). We also investigated X-ray emission due to the accretion mechanism in quiescent novae. 81 Galactic classical and recurrent novae were observed at quiescence, and only 11 were detected. Some of them are variable in X-rays on time scales of years; the X-ray spectra range from very soft to hard. The average X-ray luminosity is not larger than that of quiescent dwarf novae, even if quiescent novae are at least 10 times more luminous at optical wavelengths. There seems to be a missing boundary layer problem: a possible explanation is that boundary layer radiation in nova systems is emitted almost entirely in the extreme ultraviolet. There is no evidence of a large incidence of magnetic systems, either of enhanced X-ray luminosity in novae observed shortly before or after the outburst.
\end{abstract}

Key words. astronomical data bases: miscellaneous - stars: novae, cataclysmic variables - X-rays: stars

\section{Introduction}

Classical novae are cataclysmic variables (hereafter CV), close binary systems in which a white dwarf accretes matter from a Roche lobe-filling companion. This companion is often a main sequence star (with mass $<1 M_{\odot}$ ) and the known orbital periods are mostly in the range $2.5-8 \mathrm{hrs}$. Recurrent novae, so called because of recurrent outbursts every 10-30 years, are a class with only 10 known systems. They can be either CV or symbiotic-like systems, with a giant secondary and an orbital period of the order of a year. Both types of novae, which we will call hereafter with this comprehensive name, undergo outbursts of amplitude $\Delta m=8-15 \mathrm{mag}$ in the optical range, with the recurrent novae outbursts in the lower tail of the distribution. Novae are the second most energetic phenomenon in the Galaxy: the total energy emitted is $10^{44}-10^{46} \mathrm{erg}$. The outbursts are thought to be triggered by a thermonuclear runaway in the hydrogen burning shell at the bottom of the accreted layer. Even if there is no subsequent shock

Send offprint requests to: M. Orio,

e-mail: orio@cow.physics.wisc.edu wave a radiation driven wind follows, depleting all or part of the accreted envelope.

$\mathrm{X}$-ray emission in the ROSAT wavelength range is expected because of the following mechanisms:

1. Shortly after the outburst, residual hydrogen burning occurs in a shell on the white dwarf, if part of the accreted envelope is not ejected. The photosphere shrinks while the effective temperature increases, hydrogen burns in a shell and the post-nova appears as a very hot blackbody-like object at effective temperatures $2.5-10 \times 10^{5} \mathrm{~K}$, with $L_{\mathrm{x}} \simeq 10^{38} \mathrm{erg} \mathrm{s}^{-1}$ (the Eddington luminosity for a $1 M_{\odot}$ star; see Prialnik 1986). The post-nova at this point is a super-soft $X$-ray source, and it remains such for a time which is directly proportional to the leftover envelope mass (e.g. Kato 1997). The minimum pressure necessary for the outburst to be triggered (see Fujimoto 1982) is:

$P_{\mathrm{outb}}=\frac{G M_{\mathrm{WD}} \Delta M_{\mathrm{env}}}{4 \pi R_{\mathrm{WD}}^{4}}$

where $\Delta M_{\text {env }}$ is the mass of the accreted envelope, and $M_{\mathrm{WD}}$ and $R_{\mathrm{WD}}$ are the mass and radius of the white dwarf (WD), respectively. The higher the mass of the white 
dwarf, the earlier this pressure is reached: less mass $\Delta M_{\mathrm{env}}$ is accreted on a massive white dwarf where it is concentrated in a smaller surface. Therefore $\Delta M_{\mathrm{env}} \propto \frac{1}{M_{\mathrm{WD}}}$. Assuming that $\Delta M_{\text {env }}$ is a fixed fraction of the accreted envelope, the time to burn its hydrogen content is inversely proportional to the original white dwarf mass.

If not all the accreted material is ejected in the outburst, and if the white dwarf mass is high (as it is thought to be often the case, since outbursts on small mass WD take a much longer time to trigger and are rarer) the mass increases towards the Chandrasekhar limit after repeated outbursts. Eventually, the system undergoes a type I supernova event, or a neutron star is formed by accretion induced collapse. The model predictions are very parameter-dependent and sometimes contradictory (see Prialnik 1986; Kato 1997). The large super-soft X-ray flux of the luminous remnant, even of a few $10^{-7} \mathrm{erg} \mathrm{cm}^{-2} \mathrm{~s}^{-1}$ for Galactic novae (e.g. Balman et al. 1998; Orio 1999) is the only clear observational evidence of how long the hydrogen rich fuel lasts, therefore of how much is left after each outburst. Despite the large interstellar absorption in this energy range, super-soft X-ray source are indeed easily detected in the Magellanic Clouds, in M 31 and sometimes in the Galaxy (see Greiner 2000).

2. During the outburst, the X-ray flux can be produced by shocks in the hot circumstellar material (Brecher et al. 1977). Even if there is no shock wave in the outburst, shocks may originate in interacting winds or interaction between the ejecta and pre-existing material. It has been suggested that a high velocity wind collides into a lower velocity wind emitted in a previous stage (models are developed in Lloyd et al. 1994, 1997; O'Brien et al. 1994; O'Brien \& Lloyd 1994). The expected spectrum is thermal. The plasma temperature can be $k T=0.2-15 \mathrm{keV}$, depending on how much time has elapsed since the shock, and how efficient the cooling is. Recurrent novae have a secondary giant star, which has lost a significant amount of material through a wind. This material is heated and shocked by the nova ejecta. Hard X-ray emission attributed to shocked gas was first detected in EXOSAT observations of the recurrent nova RS Oph in its 1985 outburst (see Mason et al. 1987; Contini et al. 1995). For classical novae, shocks are suspected to occur because they explain the presence of coronal lines in the nova spectra in the optical (Williams 1992) and infrared (Gehrz et al. 1990; Benjamin \& Dinerstein 1990).

3. When novae return to quiescence, accretion phenomena produce X-rays. For the nova theory, it is critical whether accretion is rekindled, shortly after the outburst, at a high rate due to the irradiation of the hot $\mathrm{WD}$, and whether it is enhanced again before the outburst. This has been predicted by the "hibernation theory" for classical novae (Shara et al. 1986). The X-ray spectrum of nonmagnetic, non-nova $\mathrm{CV}$ is fitted with a model at plasma temperatures $k T \simeq 2 \mathrm{keV}$ (e.g. Richman 1996). Harder components may also exist. In the standard theory of disk accretion, the boundary layer is expected to re-radiate a significant faction of the gravitational energy. How large is this fraction? For other non magnetic CV, Vrtilek et al. (1994) estimate it to be about 1/4, and Richman's (1996) results are in agreement. However, van Teeseling et al. (1996) and Verbunt et al. (1997) argued instead that the fraction of re-radiated energy would be so low that the boundary layer model fails to reproduce the X-ray observations. According to these authors the X-ray luminosity is also strongly correlated with the inclination, indicating a small size emitting region, closer to the white dwarf than the boundary layer. Observing quiescent novae in X-rays is more difficult: $\mathrm{CV}$ are usually discovered in outburst and novae are at least a factor of 100 more luminous than dwarf novae, so a selection effect exists in the average distance.

We search also for possible magnetic systems among novae, because a high magnetic field may have important consequences in the mass ejection process during outburst (see Orio et al. 1992; Lepine et al. 1999). If a nova WD accretes only through the polar caps (i.e. the system is a polar) it is expected to show high plasma temperatures, even 20-30 keV (see Cropper 1990; Patterson 1994). For polar systems, there is a very soft black-body like component. Often only this component is detected (e.g. Ramsay et al. 1995). Even intermediate polars (IP), however, may exhibit a very soft component (e.g. Burwitz $\&$ Reinsch 2000). The X-ray luminosity of non-magnetic systems is on average at least an order of magnitude less than that of magnetic systems $\left(10^{30}-10^{31} \mathrm{erg} \mathrm{s}^{-1}\right.$ compared to $10^{32}-10^{33} \mathrm{erg} \mathrm{s}^{-1}$, e.g. Verbunt et al. 1997). Only few X-ray observations of quiescent accreting novae have been published so far. Einstein IPC detections of a handful of quiescent novae were described by Becker \& Marshall (1981); Drechsel et al. (1987) discussed V603 Aql. We also reported on the few detections of quiescent novae in the ROSAT All-Sky Survey (Orio et al. 1992), and analysed ROSAT pointed observations of RS Oph and CP Pup (Orio 1993; Balman et al. 1995). Other groups have studied RR Tel (Jordan et al. 1994), and mentioned the ROSAT detections of four other novae in papers dedicated to dwarf novae (Vrtilek et al. 1994; Richman 1996; van Teeseling et al. 1996; Verbunt et al. 1997). In this article, we examine the ROSAT observations of all accreting novae for a comprehensive view.

\section{The observations}

ROSAT brought important changes to X-ray astronomy. It performed the first All-Sky Survey in soft $\mathrm{X}$-rays and gave the scientific community access to a database of more than 10000 deep pointed observations, covering almost $15 \%$ of the sky and containing a very large number of serendipitously observed sources. The total number of sources in the web catalogs amounts to 95331 (see the ROSAT Consortium web page, http://wave.xray.mpe.mpg.de/rosat). More than half of these sources were not previously known, and a non negligible fraction was observed more than once. Most observations used in this work were done with the Position 
Sensitive Proportional Counter, or PSPC, sensitive in the range $0.2-2.4 \mathrm{keV}$, with a moderate spectral resolution $\left(\Delta E / E=0.43\left(E / 0.93^{-0.5}\right)\right)$ and a spatial resolution of about 25 arcsec at $1 \mathrm{keV}$. The field of view of the PSPC was 2 degrees. A few observations were also done with the HRI (High Resolution Imager), an instrument without spectral resolution but with a good spatial resolution, of about 2 arcsec, and a 38 arcmin (on a side) square field of view. The exposures in the All-Sky Survey were very short and the sensitivity limited, therefore we searched all pointed and serendipitous observations of classical novae in the ROSAT archive (partial results were given in Ögelman \& Orio 1995; Orio \& Ögelman 1996, 1998 and Orio 1999). 15 novae were targets of pointed observations; only two of them while still in the nebular phase, and four of them were observed only a few years after the outburst. Since novae are mainly observed towards the centre of the Galaxy, the number of serendipitous observations was large: we found archival exposures for 68 other Galactic novae. Also 20 novae were repeatedly observed in the Magellanic Clouds. The whole sample of 103 objects includes also 4 recurrent novae. The vignetting corrected count rates or the $3 \sigma$ upper limits for the Galactic novae observed with the ROSAT PSPC are given in Table 1. We also list basic information on the nature of the nova, if this is known: $E(B-V)$, the distance, and the time to decay by 3 mag from visual maximum, $t_{3}$ (which is correlated with the ejecta velocity, see Della Valle \& Livio 1995. These authors actually show that the time for a decay by $2 \mathrm{mag}$, is even more significant. However it is not known for a meaningful number of novae). We include the off-axis angle $\alpha$ from the centre of the PSPC and the time elapsed between the outburst and the ROSAT observation. We caution that $E(B-V)$, from which the column of neutral hydrogen is inferred (we follow Diplas \& Savage 1994), was often measured only during outburst and therefore it includes the contribution of the ejected shell in the first months, while it was still partially optically thick (e.g. Orio et al. 1996). Table 2 indicates the results for novae repeatedly observed in the Magellanic Clouds. The data analysis was performed with the MIDAS-EXSAS package (Zimmermann et al. 1994). We determined the count rates and the upper limits with the source detection and upper limits algorithm, which also corrects for the presence of the support structure. In the following sections we also make comparisons with observations of other X-ray satellites. We specially refer to the Einstein IPC (Imaging Proportional Counter), which was sensitive in the 0.4$4.0 \mathrm{keV}$ range, had spectral resolution, a 1 arcmin spatial resolution, and a field of view of 75 arcmin. Another satellite used to observe a few novae was the non-imaging EXOSAT, with the low energy (LE1) telescope and the Channel Multiplier Array (CMA), sensitive to the 0.03$2.5 \mathrm{keV}$ region without spectral resolution, a $2^{\circ}$ field of view and 18 arcsec spatial resolution. We indicate approximate conversions of count rates with different satellites using the software PIMMS, available at the NASA-Goddard Space Flight centre website. PIMMS accounts for the dif- ferent wavelength band-passes. Of course, this conversion carries uncertainties, and it is done only when a physically meaningful spectral model can be reasonably assumed.

\section{The missing super-soft $X$-ray source}

Only 3 super-soft X-ray sources are detected among the 132 Galactic and Magellanic Clouds novae observed with ROSAT. 1974 Cyg and GQ Mus are already well known super-soft sources (see Introduction, Table 1, and references therein). In the course of this project we serendipitously discovered also N LMC 1995 (see Orio \& Greiner 1999). Altogether, 30 of the novae in the Galaxy and 9 among the 25 in the Magellanic Clouds were observed within 10 years after the outburst. After the ROSAT mission, two more classical novae and one recurrent nova were observed with BeppoSAX and Chandra and found to be super-soft X-ray sources (Kahabka et al. 1999; Orio et al. 1999a). Even including these three, only $\simeq 15 \%$ of observed post-novae were detectable super-soft X-ray sources, at least for a significant length of time to be "caught" as such. Even if $E(B-V)$ is not known for the majority of the novae, the position in the Galaxy and the magnitudes reached indicate it is unlikely that $E(B-V)>0.45$ for most novae. With this value, GQ Mus was even detected in a short Survey exposure (Orio et al. 1992a). There is only one detection in the Magellanic Clouds, yet 12 other, non-nova super-soft X-ray sources, have been observed in the LMC and in the SMC (see Kahabka \& van den Heuvel 1997; Greiner 2000). Hot post-nova white dwarfs, like GQ Mus or V1974 Cyg, are therefore relatively rare.

The $3 \sigma$ upper limits on $T_{\mathrm{BB}}$ (equivalent blackbody temperature), for the novae with known $E(B-V)$ and distance are $T_{\mathrm{BB}}<20-35 \mathrm{eV}$ for $L_{\mathrm{bol}} \geq 0.1 L_{\mathrm{Edd}}$. At the $1 \sigma$ confidence level, $T_{\mathrm{BB}}<25 \mathrm{eV}$ in all observations. This is much lower than the peak temperature of a shellhydrogen- burning white dwarf with $M_{\mathrm{WD}} \geq 0.8 M_{\odot}$ calculated in several papers (e.g. Prialnik 1986; MacDonald \& Vennes 1991). It is unlikely that all observed novae have lower white dwarf mass, or that all have been observed already during turn off, while the effective temperature was already decreasing. Turn off in the UV range is also observed to occur within a few years range (GonzalezRiestra et al. 1998). There is no trace of residual supersoft X-ray emission from QU Vul and PW Vul, observed to be bright in X-rays in 1987 with EXOSAT (Ögelman et al. 1987). V1500 Cyg, for which "an almost $3 \sigma$ detection" with the Einstein IPC was reported by Chlebowski \& Kaluzny (1988) is not detected, not even in the $1-2.4 \mathrm{keV}$ range (Verbunt et al. 1997, mention a marginal detection in this range in the All-Sky Survey). A firm $3 \sigma$ upper limit is $T_{\mathrm{BB}} \leq 26 \mathrm{eV}$ assuming $L_{\mathrm{x}} \geq 10^{36} \mathrm{erg} \mathrm{s}^{-1}$ and $N(\mathrm{H}) \simeq 10^{21} \mathrm{~cm}^{-2}$ (compatible with $E(B-V)=0.69$, Wu \& Kester 1977).

In Table 5 we summarize the observations of GQ Mus, the only long term X-ray light curve, including an unpublished observation of 1994 . We give count rates, effective temperature and bolometric luminosity derived with 
Table 1. Observations of Galactic novae with the ROSAT PSPC: time elapsed between outburst and observations in years or in days $(\mathrm{d})$ and months $(\mathrm{m}), E(B-V)$, distance, time for decay from visual maximum by 3 magnitudes, ROSAT PSPC count rate $\pm 1 \sigma$ or $3 \sigma$ upper limits, image reference number in the HEASARC archive, off-axis angle $\alpha$ if the nova was not observed on axis, reference for $\mathrm{d}$ and $E(B-V)$.

\begin{tabular}{|c|c|c|c|c|c|c|c|c|}
\hline Nova & $\begin{array}{c}\text { time after } \\
\text { outburst }\end{array}$ & $E(B-V)$ & $\begin{array}{c}\text { distance } \\
(\mathrm{kpc})\end{array}$ & $\begin{array}{l}t_{3} \\
\text { (days) }\end{array}$ & $\begin{array}{c}\text { count rate } \\
\left(\operatorname{cts~s}^{-1}\right)\end{array}$ & $\begin{array}{l}\text { Image } \\
\text { number }\end{array}$ & $\begin{array}{c}\alpha \\
(\operatorname{arcmin})\end{array}$ & Ref. \\
\hline \multirow[t]{5}{*}{1974 Cyg 1992} & $4-22 \mathrm{~m}$ & $0.18-0.32$ & $2-3$ & 40 & up to $76.50 \pm 0.17$ & all & $(1,2)$ & \\
\hline & $63 \mathrm{~d}$ & & & & $0.0208 \pm 0.0031$ & 141856 & & \\
\hline & $91 \mathrm{~d}$ & & & & $0.1426 \pm 0.0110$ & 18000 & & \\
\hline & $97 \mathrm{~d}$ & & & & $0.1782 \pm 0.0071$ & 18000 & & \\
\hline & $147 \mathrm{~d}$ & & & & $0.3516 \pm 0.0122$ & 18000 & & \\
\hline 4157 Sgr 1992a & $19 \mathrm{~m}$ & $0.41-0.51$ & & & $\leq 0.0153$ & 400396 & 39 & (3) \\
\hline 351 Pup 1991 & $16 \mathrm{~m}$ & $0.3 \pm 0.1$ & $4.7 \pm 0.6$ & 40 & $0.22 \overline{3} 0 \pm 0.0005$ & 300212 & & (4) \\
\hline \multirow[t]{3}{*}{838 Her 1991} & $6 \mathrm{~d}$ & 0.53 & 3.4 & 5 & $0.1567 \pm 0.0115$ & 160060 & & $(5)$ \\
\hline & $12 \mathrm{~m}$ & & & & $\leq 0.0027$ & 300162 & & \\
\hline & $19 \mathrm{~m}$ & & & & $0.0009 \pm 0.0003$ & 300183 & & \\
\hline 868 Cen 1991 & $17 \mathrm{~m}$ & 1.7 & & & $\leq 0.0035$ & 500201 & 29 & \\
\hline 4160 Sgr 1990 & $26 \mathrm{~m}$ & & & & $\leq 0.0046$ & 200916 & 37 & $(3)$ \\
\hline \multirow[t]{2}{*}{3890 Sgr $1990(\mathrm{RN})$} & $11 \mathrm{~m}$ & 0.5 & & 10 & $\leq 0.0051$ & 400168 & 36 & (6) \\
\hline & $17 \mathrm{~m}$ & & & & $\leq 0.0013$ & 400310 & 22 & \\
\hline \multirow[t]{2}{*}{443 Sct 1989} & $30 \mathrm{~m}$ & 0.41 & 8 & $46 \pm 9$ & $\leq 0.0042$ & 300124 & 30 & $(6,7)$ \\
\hline & 3 & & & & $\leq 0.0037$ & 400316 & 27 & \\
\hline \multirow[t]{3}{*}{745 Sco 1989 (RN) } & 2.5 & 1.1 & & & $\leq 0.0031$ & 900198 & 53 & \\
\hline & 2.5 & & & & $\leq 0.0022$ & 400151 & 53 & $(7)$ \\
\hline & 3.5 & & & & $\leq 0.0030$ & 400269 & 53 & \\
\hline 394 CrA 1987 (RN) & $19.5 \mathrm{~m}$ & & & 10 & $\overline{\leq} 0.0011$ & 300042 & & $(8)$ \\
\hline \multirow[t]{2}{*}{ QV Vul 1987} & 3.25 & 0.32 & $4.5-4.7$ & 60 & $\leq 0.0024$ & 300040 & & (7) \\
\hline & 4.25 & & & & $\leq 0.0034$ & $300040-1$ & & \\
\hline \multirow[t]{2}{*}{827 Her 1987} & $50 \mathrm{~m}$ & & 2.64 & 57 & $\leq 0.0030$ & 300041 & & \\
\hline & $62 \mathrm{~m}$ & & & & $\leq 0.0033$ & $300041-1$ & & \\
\hline OS And 1986 & 5 & $0.26 \pm 0.04$ & 5.1 & 22 & $\leq 0.0008$ & 300039 & & (9) \\
\hline Sgr 1986 & 6 & & & & $\leq 0.0033$ & 201093 & 36 & \\
\hline QU Vul 1984 & 5.3 & 0.57 & $2.6 \pm 0.2$ & 34 & $0.0034 \pm 0.0007$ & 300037 & & (10) \\
\hline \multirow[t]{2}{*}{ PW Vul 1984} & 6.9 & 0.55 & $1.6 \pm 0.2$ & 147 & $\leq 0.0038$ & 300036 & & (11) \\
\hline & 8.9 & & & & $\leq 0.0020$ & 300036-1 & & \\
\hline \multirow[t]{6}{*}{4092 Sgr 1984} & 7.5 & & & & $\leq 0.0035$ & 400209 & 17 & \\
\hline & 7.5 & & & & $\leq 0.0100$ & 400201 & 33 & \\
\hline & 7.5 & & & & $\leq 0.0113$ & 400202 & 45 & \\
\hline & 7.5 & & & & $\leq 0.0130$ & 400210 & 36 & \\
\hline & 7.5 & & & & $\leq 0.0119$ & $400211-1$ & 55 & \\
\hline & 7.5 & & & & $\leq 0.0249$ & 400211 & 55 & \\
\hline \multirow[t]{4}{*}{ GQ Mus 1983} & 9 & $0.45 \pm 0.15$ & $48 \pm 1$ & 45 & $0.1137 \pm 0.0046$ & 300035 & & $(12)$ \\
\hline & 10 & & & & $0.0066 \pm 0.0014$ & 300336 & & \\
\hline & 10.8 & & & & $\leq 0.0015$ & 300336 & & \\
\hline & 11.6 & & & & $\leq 0.0019$ & $300336-1$ & & \\
\hline MU Ser 1983 & 10 & & & 5 & $\leq 0.0012$ & 900170 & & \\
\hline \multirow[t]{2}{*}{ RS Oph 1985 RN } & 6 & 0.70 & $1.2-2$ & & $0.0035 \pm 0.0010$ & 300038 & 7 & (6) \\
\hline & 7 & & & & $0.0116 \pm 0.0017$ & $300038-1$ & & \\
\hline 4121 Sgr 1983 & 10 & & & & $\leq 0.0017$ & 201093 & 41 & \\
\hline SS LMi 1980 & 12 & & & & $\leq 0.0098$ & 700433 & 40 & \\
\hline 4065 Sgr 1980 & 14 & & & & $\overline{\leq} 0.0093$ & 500009 & 50 & \\
\hline \multirow[t]{2}{*}{ HS Sge 1977} & 15 & & & 20 & $\leq 0.0057$ & 500190 & 50 & \\
\hline & 15 & & & & $\leq 0.0079$ & 500191 & 48 & \\
\hline 2104 Oph 1976 & 26 & & & & $\leq 0.0083$ & 800394 & 52 & \\
\hline NQ Vul 1976 & 25 & 0.37 & 2.1 & 65 & $\leq 0.0077$ & 170260 & 56 & \\
\hline \multirow[t]{3}{*}{1500 Cyg 1975} & 26 & 0.69 & $1.2 \pm 0.2$ & 3.6 & $\leq 0.0038$ & 300033 & & \\
\hline & 27 & & & & $\leq 0.0042$ & $30033-1$ & & \\
\hline & 27 & & & & $\leq 0.0020$ & 900194 & 47 & \\
\hline
\end{tabular}


Table 1. continued.

\begin{tabular}{|c|c|c|c|c|c|c|c|c|}
\hline Nova & $\begin{array}{c}\text { time after } \\
\text { outburst }\end{array}$ & $E(B-V)$ & $\begin{array}{c}\text { distance } \\
(\mathrm{kpc})\end{array}$ & $\begin{array}{c}t_{3} \\
\text { (days) }\end{array}$ & $\begin{array}{c}\text { count rate } \\
\left(\operatorname{cts~s}^{-1}\right)\end{array}$ & $\begin{array}{l}\text { Image } \\
\text { number }\end{array}$ & $\begin{array}{c}\alpha \\
(\operatorname{arcmin})\end{array}$ & Ref. \\
\hline 1301 Aql 1975 & 26 & & & 35 & $\leq 0.0033$ & 200126 & 26 & \\
\hline \multirow[t]{5}{*}{ Car 1972} & 20 & & & & $\leq 0.0120$ & 200851 & 42 & \\
\hline & 20 & & & & $\leq 0.0053$ & 200852 & 23 & \\
\hline & 20 & & & & $\leq 0.0230$ & 200857 & 37 & \\
\hline & 20 & & & & $\leq 0.0280$ & 200866 & 42 & \\
\hline & 20 & & & & $\leq 0.0057$ & 200867 & 22 & \\
\hline \multirow[t]{2}{*}{ Car 1972} & 21 & & & & $\leq 0.0185$ & $200872-1$ & 36 & \\
\hline & 21 & & & & $\leq 0.0232$ & 200872 & 36 & \\
\hline 1330 Cyg 1970 & 21 & & & 18 & $\leq 0.0064$ & 400138 & 46 & \\
\hline \multirow[t]{3}{*}{ LV Vul 1968} & 24 & 0.40 & 2.1 & 37 & $\leq 0.0079$ & 500086 & 33 & \\
\hline & 24 & & & & $\leq 0.0097$ & 500083 & 33 & \\
\hline & 24 & & & & $\leq 0.0237$ & 500087 & 33 & \\
\hline \multirow[t]{2}{*}{655 CrA 1967} & 24 & & & & $\leq 0.0020$ & 400033 & 14 & \\
\hline & 25 & & & & $\leq 0.0021$ & $400033-1$ & 14 & \\
\hline \multirow[t]{2}{*}{ NSV09828 Sgr 1963} & 29 & & & 12 & $\leq 0.0100$ & 400209 & 37 & \\
\hline & 29 & & & & $\leq 0.0103$ & 400210 & 49 & \\
\hline 592 Her 1962 & 30 & & & 27 & $\leq 0.0032$ & 201228 & 16 & \\
\hline AL Com 1961 & 30 & & & 30 & $\leq 0.0035$ & 700346 & 54 & \\
\hline \multirow{2}{*}{366 Sct 1961} & 30 & & & & $\leq 0.0105$ & 500040 & 37 & \\
\hline & 30 & & & & $\leq 0.0014$ & 500049 & 50 & \\
\hline \multirow[t]{3}{*}{446 Her 1960} & 33 & 0.037 & 1.38 & 16 & $0.0079 \pm 0.0112$ & 600282 & 36 & \\
\hline & 33 & & & & $0.0119 \pm 0.0152$ & 600282 & 36 & \\
\hline & 33 & & & & $0.0080 \pm 0.0113$ & 600283 & 35 & \\
\hline 972 Oph 1957 & 35 & & & 176 & $\leq 0.0051$ & 900204 & 47 & \\
\hline 1275 Sgr 1954 & 36 & & & $>10$ & $\leq 0.0033$ & 200710 & 18 & \\
\hline 723 Sco 1952 & 41 & & & 17 & $\leq 0.0012$ & 201383 & 49 & \\
\hline \multirow{9}{*}{2415 Sgr 1951} & 41 & & & & $\leq 0.0101$ & 400210 & 49 & \\
\hline & 41 & & & & $\leq 0.0074$ & 400201 & 25 & \\
\hline & 41 & & & & $\leq 0.0089$ & 400202 & 45 & \\
\hline & 41 & & & & $\leq 0.0113$ & 400187 & 45 & \\
\hline & 41 & & & & $\leq 0.0150$ & 400195 & 31 & \\
\hline & 41 & & & & $\leq 0.0123$ & 400196 & 48 & \\
\hline & 41 & & & & $\leq 0.0120$ & 400209 & 33 & \\
\hline & 41 & & & & $\leq 0.0089$ & 400202 & 45 & \\
\hline & 41 & & & & $\leq 0.0092$ & 400195 & 31 & \\
\hline \multirow[t]{3}{*}{720 Sco 1950} & 43 & & & & $\leq 0.0010$ & 201383 & 42 & \\
\hline & 42 & & & & $\leq 0.0010$ & 200983 & 42 & \\
\hline & 43 & & & & $\leq 0.0008$ & $200983-1$ & 42 & \\
\hline \multirow[t]{2}{*}{902 Sco 1949} & 44 & & & 200 & $\leq 0.0227$ & 500199-1 & 43 & \\
\hline & 43 & & & & $\leq 0.0051$ & 500199 & 43 & \\
\hline \multirow[t]{2}{*}{ RR Tel 1948} & 44 & 0.08 & 2.6 & $>2000$ & $0.1666 \pm 0.0060$ & 200581 & & $(13,14)$ \\
\hline & 45 & & & & $0.2297 \pm 0.0104$ & 300185 & 19 & \\
\hline 1431 Sgr 1947 & 45 & & & & $\leq 0.0017$ & 600418 & 18 & \\
\hline 1150 Sgr 1946 & 45 & & & $<600$ & $\leq 0.0042$ & 500009 & 21 & \\
\hline 1431 Sgr 1945 & 48 & & & & $\leq 0.0017$ & 600418 & 18 & \\
\hline 500 Aql 1943 & 49 & & & 42 & $\leq 0.0021$ & 200898 & 34 & \\
\hline 1148 Sgr 1943 & 50 & & & & $\leq 0.0104$ & 400396 & 31 & \\
\hline CP Pup 1942 & 50 & $0.1-0.25$ & 0.7 & 8 & $0.0652 \pm 0.0028$ & 300212 & & $(15)$ \\
\hline 450 Cyg 1942 & 49 & & & 108 & $\leq 0.0036$ & 400138 & 52 & \\
\hline \multirow[t]{2}{*}{ BT Mon 1939} & 54 & & 5.3 & $36 ?$ & $\leq 0.0095$ & $900266-1$ & 32 & \\
\hline & 54 & & & & $\leq 0.0080$ & 900266 & 32 & \\
\hline CP Lac 1936 & 57 & 0.48 & 1.15 & 10 & $0.0037 \pm 0.0011$ & 20128 & 6 & \\
\hline DQ Her 1934 & 58 & 0.07 & 0.33 & 94 & $0.0121 \pm 0.0011$ & 300103 & & \\
\hline 737 Sgr 1933 & 60 & & & $>70$ & $\leq 0.0047$ & 201093 & 31 & \\
\hline 1014 Sgr 1933 & 60 & & & $>50$ & $\leq 0.0023$ & 201093 & 52 & \\
\hline
\end{tabular}


Table 1. continued.

\begin{tabular}{|c|c|c|c|c|c|c|c|}
\hline Nova & $\begin{array}{c}\text { time after } \\
\text { outburst }\end{array}$ & $E(B-V)$ & $\begin{array}{c}\text { distance } \\
(\mathrm{kpc})\end{array}$ & $\begin{array}{c}t_{3} \\
\text { (days) }\end{array}$ & $\begin{array}{c}\text { count rate } \\
\left(\text { cts s }^{-1}\right)\end{array}$ & $\begin{array}{l}\text { Image } \\
\text { number }\end{array}$ & $\begin{array}{cc}\alpha & \text { Ref. } \\
(\operatorname{arcmin}) & \end{array}$ \\
\hline 441 Sgr 1930 & 61 & & & 53 & $\leq 0.0055$ & 300050 & 51 \\
\hline HV Vir 1929 & 64 & & & & $\leq 0.0028$ & 300322 & \\
\hline 1583 Sgr 1928 & 63 & & & 37 & $\leq 0.0052$ & 50009 & 48 \\
\hline FM Sgr 1926 & 65 & & & 30 & $\leq 0.0055$ & 500009 & 25 \\
\hline KY Sgr 1926 & 66 & & & 60 & $\leq 0.0027$ & 400330 & 40 \\
\hline RR Pic 1925 & 68 & $0.02-0.07$ & 0.263 & 150 & $0.0706 \pm 0.0053$ & 300288 & \\
\hline \multirow[t]{2}{*}{ FL Sgr 1924} & 47 & & & 32 & $\leq 0.0022$ & 400151 & 53 \\
\hline & 49 & & & & $\leq 0.0029$ & 900198 & 53 \\
\hline GR Sgr 1924 & 67 & & & & $\leq 0.0058$ & 300050 & 49 \\
\hline IM Nor 1920 & 73 & & & & $\leq 0.0010$ & 400375 & 24 \\
\hline \multirow[t]{4}{*}{603 Aql 1918} & 73 & 0.07 & 0.43 & 8 & $0.3151 \pm 0.0063$ & 300056 & \\
\hline & 74 & & & & $0.7791 \pm 0.0311$ & 300235 & \\
\hline & 74 & & & & $1.3035 \pm 0.0321$ & 300247 & \\
\hline & 74 & & & & $0.7790-1.3230$ & 300255 to 300266 & \\
\hline \multirow[t]{4}{*}{ SS Sge 1916} & 76 & & & & $\leq 0.0028$ & 201100 & 41 \\
\hline & 77 & & & & $\leq 0.0247$ & $201100-1$ & 41 \\
\hline & 76 & & & & $\leq 0.0085$ & 500190 & 39 \\
\hline & 76 & & & & $\leq 0.0095$ & 500190-500191 & 38 \\
\hline GR Ori 1916 & 75 & & & & $\leq 0.0024$ & 200040 & 40 \\
\hline GL Sct 1915 & 77 & & & & $\leq 0.0017$ & 200183 & 51 \\
\hline \multirow[t]{2}{*}{711 Sco 1906} & 87 & & & & 0.0020 & 200983-1 & 28 \\
\hline & 87 & & & & $\leq 0.0018$ & 201383 & 34 \\
\hline \multirow[t]{3}{*}{382 Sco 1901} & 92 & & & & $\leq 0.0009$ & 200983 & 44 \\
\hline & 92 & & & & $\leq 0.0010$ & 200983-1 & 44 \\
\hline & 92 & & & & $\leq 0.0010$ & 201383 & 36 \\
\hline GK Per 1901 & 92 & $0.07-0.29$ & 0.575 & 13 & $0.2131 \pm 0.0038$ & 300217 & 41 \\
\hline AT Sgr 1900 & 93 & & & & $\leq 0.0135$ & 400369 & 50 \\
\hline 384 Sco 1893 & 99 & & & & $\leq 0.0065$ & 200710 & 35 \\
\hline T Sco 1860 & 20 & 0.35 & & 21 & $0.0040 \pm 0.0008$ & 300113 & \\
\hline T Boo 1860 & 130 & & & & $\leq 0.0010$ & 150018 & 24 \\
\hline WY Sge 1793 & 199 & 0.6 & & & $<0.0015$ & 500209 & 46 \\
\hline \multirow[t]{3}{*}{ CK Vul 1670} & 332 & 0.73 & 2.1 & & $\leq 0.0041$ & 50086 & 25 \\
\hline & 332 & & & & $\leq 0.0050$ & 50083 & 25 \\
\hline & 332 & & & & $\leq 0.0082$ & 50087 & 25 \\
\hline
\end{tabular}

a References: (1) Mathis et al. (1995) and references therein, (2) Krautter et al. (1996) and references therein, (3) Williams (1994), (4) Orio et al. (1996), (5) Lynch et al. (1992), (6) Anupama \& Mikolawjeska (1999), (7) Anupama et al. (1992), (8) Sekiguchi et al. (1989), (9) Schwarz et al. (1997), (10) Della Valle et al. (1997), (11) Saizar et al. (1992); Ringwald \& Naylor (1996) (12) Ögelman et al. (1991) and references therein, (13) Jordan et al. (1994), (14) Whitelock (1988), (15) MacLaughlin (1960). Other estimates of distances and absorption are taken from Harrison 85 Gehrz (1988) and references therein; we caution that distance estimates given in this work based on the value of $t_{3}$ are highly uncertain. Values of $t_{3}$ are from Dürbeck (1988), unless other reference is given.

two different models, including a $\mathrm{C}-\mathrm{O}$ white dwarf atmospheric model in LTE by MacDonald \& Vennes (1991), not used in the detection papers. Atmospheric models yield lower values of the luminosity and higher $T_{\mathrm{WD}}$ than the blackbody assumption. The model was studied for a white dwarf of $1.2 M_{\odot}$ emitting at Eddington luminosity $\left(L_{\mathrm{bol}}=\right.$ $\left.1.6 \times 10^{38} \mathrm{erg} \mathrm{s}^{-1}\right)$, and we assume $d=4.75 \pm 1.55 \mathrm{kpc}$ (Krautter \& Williams 1989). The bolometric luminosity is expected to vary by up to a factor of 10 between 1.3 and $0.6 M_{\odot}$. It is commonly re-scaled with the mass using the formula $L_{\mathrm{bol}}=A\left(M_{\mathrm{WD}}-B\right)$, where $B$ is a function of the chemical composition of the envelope, and ranges between 0.2 and 0.6 (e.g. Truran 1979).

The EXOSAT LE telescope did not possess spectral resolution, however the count rates of the first three observations are consistent with the predictions of a blackbody model with the parameters reported in the table. The spectrum during the 1990 All-Sky Survey observation was very soft (Orio et al. 1992a), consistently with a blackbody temperature in the range $30-50 \mathrm{eV}$. 
Table 2. Observations of LMC novae with the ROSAT PSPC and HRI: PSPC count rates or $3 \sigma$ PSPC upper limits, or HRI equivalent count rate (HRI eq). Conversion from HRI to PSPC count rates is done according to Greiner et al. 1996. The LMC 1995 count rate entered is the highest of all observations (Orio $\&$ Greiner 1999). We indicate also the number years elapsed between outburst and observations, number of exposures, the range of off-axis angles $\alpha$ in arcmin.

\begin{tabular}{|c|c|c|c|c|}
\hline Nova & $\begin{array}{l}\text { count rate } \\
\left(\operatorname{cts} \mathrm{s}^{-1}\right)\end{array}$ & $\begin{array}{l}\text { Years } \\
\text { after }\end{array}$ & $\begin{array}{l}\text { number } \\
\text { of times }\end{array}$ & $\alpha$ \\
\hline LMC 1926 & $\leq 0.0034$ & $64-67$ & 3 & $33-40$ \\
\hline LMC 1936 & $\leq 0.0024$ & $54-57$ & 3 & $40-46$ \\
\hline LMC 1948 & $\leq 0.0023$ & $42-50$ & 7 & $20-45$ \\
\hline LMC 1951 & $\leq 0.0011$ & $39-42$ & 1 & 9 \\
\hline LMC 1968 & $\leq 0.0016$ & 25 & 1 & 52 \\
\hline LMC 1970 & $\leq 0.0024$ & $20-28$ & 6 & $13-40$ \\
\hline LMC 1970b & $\leq 0.00$ & $21-23$ & 4 & $36-43$ \\
\hline LMC 1971a & $\leq 0.0090$ & $22-23$ & 16 & $36-43$ \\
\hline LMC $1972 ?$ & $0.0055 \pm 0.0011$ & $18-21$ & 30 & $15-46$ \\
\hline LMC 1973 & $\leq 0.0017$ & $17-20$ & 8 & $9-14$ \\
\hline LMC 1977 & $\leq 0.0097$ & $14-16$ & 5 & 47 \\
\hline LMC 1978a & $\leq 0.0025$ & $14-15$ & 2 & 48 \\
\hline LMC 1978b & $\leq 0.0034$ & $14-15$ & 1 & $37-45$ \\
\hline LMC 1981 & $\leq 0.0023$ & $9-17$ & 6 & $10-27$ \\
\hline LMC 1987 & $\leq 0.0040$ & $3-7$ & 30 & $0-22$ \\
\hline LMC 1988a & $\leq 0.0106$ & $2-10$ & 20 & $6-37$ \\
\hline LMC 1988b & $\leq 0.0051$ & $2-3.8$ & 2 & $0-8$ \\
\hline LMC 1990a & $\leq 0.00$ & $0.3-9$ & 19 & $12-47$ \\
\hline LMC 1990b & $\leq 0.0015$ & 3 & 1 & $9-33$ \\
\hline \multirow[t]{2}{*}{ LMC 1992} & (HRI eq) 0.0080 & 0.25 & 1 & $16-52$ \\
\hline & $\leq 0.0050$ & $0.1-10$ & 6 & \\
\hline LMC 1995 & $0.0610 \pm 0.0003$ & $0.3-4$ & 8 & $0-44$ \\
\hline SMC 1927 & $\leq 0.0025$ & $65-66$ & 2 & 45 \\
\hline SMC 1951 & $\leq 0.0018$ & $41-43$ & 3 & $14-43$ \\
\hline SMC 1952 & $\leq 0.0015$ & $40-41$ & 6 & $20-24$ \\
\hline SMC 1994 & $($ HRI eq $) \leq 0.0028$ & 2 & 16 & $2-40$ \\
\hline
\end{tabular}

The conversion of EXOSAT into ROSAT count rates in the super-soft range is uncertain and we do not attempt to compare the luminosity. In 1992 we were observing the "plateau" constant bolometric luminosity, because the turn-off decay of the luminosity is expected to last only about one year (Prialnik, private communication). The $3 \sigma$ range of values of the bolometric luminosity is below $0.1 L_{\text {Edd }}$ for a $1.2 M_{\odot}$ WD $\left(L_{\text {Edd }}\right.$ as given by MacDonald \& Vennes 1991; there is an additional uncertainty of a factor of 2 due to the $33 \%$ distance uncertainty). Therefore, the white dwarf mass must be significantly smaller than $1.2 M_{\odot}$, around the lowest end of the range, $0.6 M_{\odot}$. The $3 \sigma$ lower limit $T_{\mathrm{WD}} \geq 400000 \mathrm{~K}$ is also the peak temperature of a WD of $0.6 M_{\odot}$. By 1993, turn-off started because the effective temperature was too low for a postnova which is still burning hydrogen. We conclude that hydrogen burning lasted for 8 to 9 years. This is exactly the length of the constant bolometric luminosity phase for a WD mass $0.6 M_{\odot}$ according to Kato (1997), so things seem to fit together.

RR Tel, a transition object between classical and symbiotic novae, is a EUV source with a hard X-ray tail, and not a super-soft X-ray source in the conventional sense. The super-soft portion of the spectrum varies inversely with the hard X-rays flux (Fig. 1). Before repeated ROSAT observations, Jordan et al. (1994) had speculated instead that only the hard X-ray component varies. We attribute the large increase of the super-soft component, (while the counts above $0.5 \mathrm{keV}$ decreased) to a blanketing effect of the wind.

\section{The hard component after the outburst}

In this and in the next section we will use the adjective "hard" for the ROSAT range 0.8-2.4 keV. Almost all the novae in outburst during the ROSAT lifetime were observed. Four out of the seven Galactic novae observed with the PSPC within two years from the outburst showed hard X-ray emission: V838 Her (N Her 1991) (see Lloyd et al. 1992), V351 Pup (N Pup 1991), V3890 Sgr, and initially also V1974 Cyg. They represent $60 \%$ of the available targets. Six other novae (V382 Vel, PW Vul, QV Vul, RS Oph, LMC 1992 and 1995), were observed to be bright in X-rays either with the ROSAT HRI or with other satellites within two years from the outburst, although only V382 Vel was observed with spectral resolution. To these we have to add at least one LMC nova, LMC 1992, detected (see Table 2) in the fourth month post-outburst (even if undetected in the following month). Unfortunately, for the hard X-ray emission the observations in the LMC cannot add a large amount of information. At a distance $d \simeq 50 \mathrm{kpc}$, sources with luminosities $L_{\mathrm{x}} \leq 10^{34} \mathrm{erg} \mathrm{s}^{-1}$ (typical for the novae observed as hard sources with ROSAT) are detected with exposures longer than $10^{4} \mathrm{~s}$, which were rarely done.

Looking more in detail at the observation of the individual objects, it is likely that ROSAT did not cover the whole X-ray spectrum of the shocked nebula in the first few months post-outburst. This appears to be quite evident for V382 Her, observed only after 6 days, and for the observations of V1974 Cyg in the first 5 months. Fits of thermal models (available in EXSAS) to the observed spectra (6 days post-outburst for V383 Her, and 63 days for V1974 Cyg), leave undetermined whether the plasma temperature was in the $5-15 \mathrm{keV}$ range with $N(\mathrm{H}) \leq 10^{22} \mathrm{~cm}^{-2}$, or instead $k T \simeq 1-2 \mathrm{keV}$ with $N(\mathrm{H})>10^{22} \mathrm{~cm}^{-2}$. We consider the first possibility more likely, at the light of the V382 Vel BeppoSAX and ASCA observations (Orio et al. 2001; Mukai \& Ishida 2001). Of course, high $N(\mathrm{H})$ cannot be ruled out: in V382 Vel it was due to internal absorption of the ejecta, rich in $\mathrm{C}$ and $\mathrm{O}$. Probably the X-ray emission came initially from deeply inside the ejecta, or from a very clumpy material. V1974 Cyg was observed again before the super-soft X-ray emission became dominant. At day 91 the plasma temperature was not well constrained yet, and $N(\mathrm{H}) \leq 3.9 \times 10^{21} \mathrm{~cm}^{-2}$, but 
Table 3. Journal of X-ray observations of GQ Mus: vignetting corrected count rates $\pm 1 \sigma$ or $3 \sigma$ upper limits, and parameters derived from spectral fits. MDV-CO refers to the model of a CO WD atmosphere described in the text (MacDonald \& Vennes 1991).

\begin{tabular}{|c|c|c|c|c|c|c|}
\hline $\begin{array}{l}\text { Observation } \\
\text { date }\end{array}$ & Instrument & $\begin{array}{l}\text { Count rate } \\
\left(\operatorname{cts} \mathrm{s}^{-1}\right)\end{array}$ & Model & $\begin{array}{c}T_{\mathrm{WD}} \\
10^{3}(\mathrm{~K})\end{array}$ & $\begin{array}{l}\text { Luminosity } \\
\left(10^{37} \mathrm{erg} \mathrm{s}^{-1}\right)\end{array}$ & Reference \\
\hline April 1984 & EXOSAT LE1 & $0.0034 \pm 0.0009$ & & & & Ögelman et al. (1984) \\
\hline July 1984 & XOSAT LE1 & $0.0028 \pm 0.0004$ & & & & Ögelman et al. (1987) \\
\hline November 1984 & EXOSAT LE1 & $0.0034 \pm 0.0007$ & blackbody & $280 \pm 50$ & $5 \pm 5$ & Ögelman et al. (1987) \\
\hline June 1985 & EXOSAT LE1 & $0.0014 \pm 0.0004$ & & & & Ögelman et al. (1987) \\
\hline July-Aug. 1990 & ROSAT PSPC & $0.1650 \pm 0.0430$ & blackbody & & & Orio et al. (1992) \\
\hline February 1992 & ROSAT PSPC & $0.1137 \pm 0.0046$ & $\begin{array}{l}\text { MDV-CO } \\
\text { blackbody }\end{array}$ & $\begin{array}{c}430_{-23}^{+10} \\
340 \pm 12\end{array}$ & $\begin{array}{c}0.15_{-0.05}^{+1.40} \\
\geq 2.6\end{array}$ & Ögelman et al. (1993) \\
\hline January 1993 & ROSAT PSPC & $0.0066 \pm 0.0014$ & $\begin{array}{l}\text { MDV-CO } \\
\text { blackbody }\end{array}$ & $\begin{array}{c}195_{-20}^{+87} \\
250 \pm 60\end{array}$ & $>0.1$ & Shanley et al. (1995) \\
\hline $\begin{array}{l}\text { Aug.-Sept. } 1993 \\
\text { July } 1994\end{array}$ & $\begin{array}{l}\text { ROSAT PSPC } \\
\text { ROSAT PSPC }\end{array}$ & $\begin{array}{l}\leq 0.0011 \\
\leq 0.0012\end{array}$ & $\begin{array}{l}\text { blackbody } \\
\text { blackbody }\end{array}$ & $\begin{array}{l}\leq 200 \\
\leq 200\end{array}$ & & $\begin{array}{l}\text { Shanley et al. (1995) } \\
\text { this paper }\end{array}$ \\
\hline
\end{tabular}

in one observation of day 147, the plasma temperature was bound to be below $1.4 \mathrm{keV}$ ( $3 \sigma$ upper limits). The temperature was quickly cooling or the shell absorption was rapidly decreasing, or both things were happening. Also for V382 Her, Orio et al. (2001) found $k T \leq 1 \mathrm{keV}$ half a year post-outburst. As the temperature of the emitting plasma was decreasing, the total hard flux increased in the case of N Cyg 1992 in the first few months after the outburst (see also Balman et al. 1998). This could be due either to the thinning of the ejecta to X-rays, or to an increasingly large shocked region.

How long does it take for the shocked nebula to cool? V3890 Sgr, the only recurrent nova in outburst during the operation period of ROSAT, has a red giant companion and other characteristics in common with RS Oph, the first hard X-ray source observed among post-novae. This nova was detected in the ROSAT All-Sky-Survey (Orio et al. 1992a) but it did not appear as an X-ray source in serendipitous pointings after 11 and 17 months, so the emitting shell must have cooled by then. We also know that the cooling time can definitely be longer than 11 months. V351 Pup (N Pup 1991) was X-ray bright 16 months after the outburst (see also Orio et al. 1996), but the X-ray source had faded in a later observation done with ASCA in 1998 (Orio \& Mukai 2001). At 16 months the best fits of a thermal plasma were consistent with lack of intrinsic absorption, $k T \simeq 1 \mathrm{keV}$, and X-ray luminosity of a few times $10^{33} \mathrm{erg} \mathrm{s}^{-1}$. In exceptional cases, we find hard X-ray emission from the shell even tens of years after the outburst. For RR Tel, after 43 years the hard $\mathrm{X}$-ray component is ascribed to colliding winds producing a shock (Jordan et al. 1994; Contini \& Formiggini 1999). We fit the data with flux of a few $10^{-14} \mathrm{erg} \mathrm{cm}^{-2} \mathrm{~s}^{-1}$ and plasma temperatures 200 and $350 \mathrm{eV}$ in the two observations respectively. GK Per had a large amount of circum- stellar material, most likely residual of a planetary nebula phase. In the best fit with two components, we find that the total flux of the softest component is $\simeq 25 \%$ the total flux (approximately $8 \times 10^{-12} \mathrm{erg} \mathrm{cm}^{-2} \mathrm{~s}^{-1}$ ), with $k T \simeq 200 \mathrm{eV}$ and $N(\mathrm{H}) \simeq 10^{21} \mathrm{~cm}^{-2}$ (consistent with the measured value $E(B-V) \simeq 0.1)$. Balman \& Ögelman (1999) indeed found in a later, spatially resolved HRI observation, that a fraction $20-25 \%$ of the total flux is due to the extended shell and this component is the softest one.

\section{The accretion process: signatures in $\mathrm{X}$-rays}

The novae included in Table 4, except RR Tel and partially GK Per, were observed in X-rays at quiescence because of accretion. However, only 11 out of 81 quiescent Galactic novae observed in the pointed observations were detected. Only the detection of CP Lac is entirely new. Comparing catalogs of classical novae (Dürbeck 1988) and of dwarf novae (Khruzina \& Shugarov 1991) we find that classical novae are on average only $\simeq 2$ magnitude fainter at quiescence, despite a larger average distance by an order of magnitude. The X-ray luminosity of novae however, at least in the ROSAT range, is not higher than for dwarf novae. For the detected objects $L_{\mathrm{x}}=10^{30-33} \mathrm{erg} \mathrm{s}^{-1}$ (see Table 5). We notice that the novae that appear as X-ray luminous at quiescence were all fast novae in outburst. We see in Fig. 1 a complex and large variety of spectral characteristics, even in the relatively narrow ROSAT range. The spectra vary from very soft, like RR Pic, to to hard, like CP Pup. Also the ratio of $L_{\mathrm{x}} / L_{\mathrm{opt}}$ varies, as shown in Table 5.

For the non detected Galactic novae, assuming a plasma temperature $k T \simeq 1 \mathrm{keV}$ for the accretion disk, the $3 \sigma$ upper limits on the ROSAT X-ray flux are always 

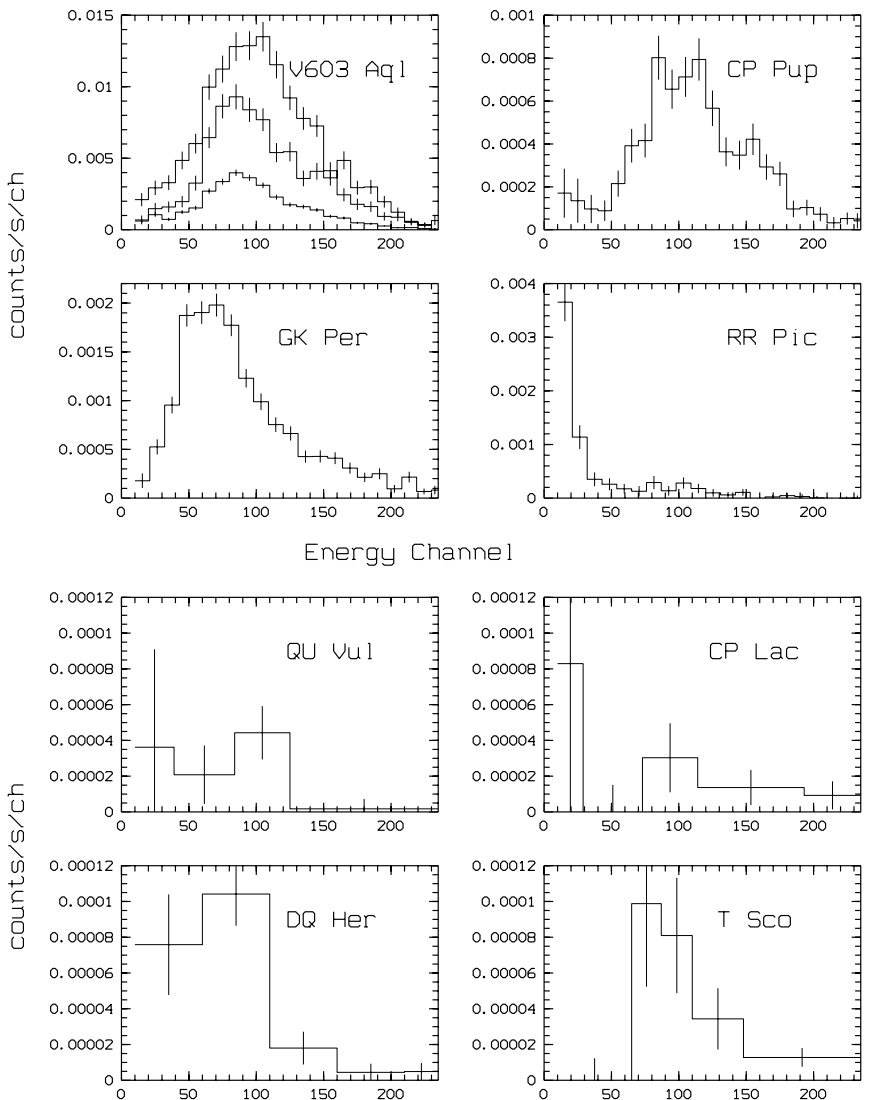

Energy Channel
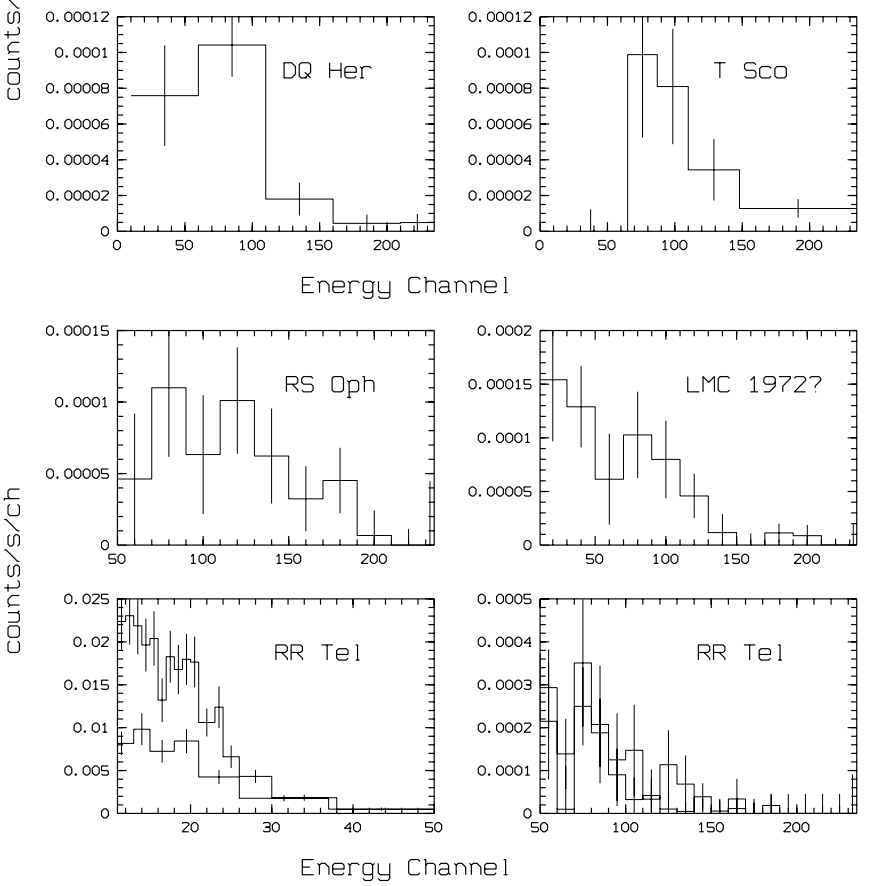

Fig. 1. ROSAT PSPC spectra of 11 quiescent novae (in two different observations for V603 Aql and RR Tel). We show the count rate per energy channel; each of the channels is approximately equivalent to $10 \mathrm{eV}$. The spectra of the brightest novae in X-rays are on top, except for the one of RR Tel which is split in two (soft and hard) in the bottom row. The identification of an X-ray source with N LMC 1972 is uncertain due to large off-axis angles and a crowded field.

below a few $10^{-13} \mathrm{erg} \mathrm{cm}^{-2} \mathrm{~s}^{-1}$. At an average distance $1-2 \mathrm{kpc}$ this corresponds to $L_{\mathrm{x}} \leq 10^{30} \mathrm{erg} \mathrm{s}^{-1}$. Since most IP are at higher X-ray luminosity and exhibit a hard $\mathrm{X}$-ray flux component, which is not significantly affected by interstellar absorption, this is a general indication that IP do not seem to be very frequent among novae (we caution however that DQ Her, the prototype of intermediate
Table 4. X-ray observations of quiescent novae: count rates or $3 \sigma$ upper limits, and PSPC hardness ratio h.r. (count rate at $0.20-0.50 \mathrm{keV} /$ count rate at $0.51-2.40 \mathrm{keV}$, if measurable with less than $10 \%$ uncertainty). For the IPC the approximate $\mathrm{PSPC}$ equivalent count rate is in parenthesis.

\begin{tabular}{|c|c|c|c|}
\hline Nova & Instrument & c.r. & h.r. \\
\hline \multicolumn{4}{|l|}{ RR Tel } \\
\hline 1978 & Einstein IPC & $0.018 \pm 0.005(0.06)$ & \\
\hline 1990 & PSPC (Survey) & $0.312 \pm 0.053$ & \\
\hline 1992 & PSPC & $0.167 \pm 0.006$ & \\
\hline 1993 & PSPC & $0.230 \pm 0.010$ & $>4$ \\
\hline \multicolumn{4}{|l|}{ QU Vul } \\
\hline 1991 & PSPC & $0.003 \pm 0.001$ & 0.32 \\
\hline \multicolumn{4}{|l|}{ RR Pic } \\
\hline 1979 & Einstein IPC & $0.031 \pm 0.005(0.10)$ & \\
\hline 1990 & PSPC (Survey) & $0.144 \pm 0.043$ & \\
\hline 1992-1993 & PSPC & $0.071 \pm 0.005$ & 3.10 \\
\hline \multicolumn{4}{|l|}{ V603 Aql } \\
\hline 1981 & Einstein IPC & $0.71 \pm 0.03(1.30)$ & \\
\hline 1983 & Einstein IPC & $0.279 \pm 0.013(0.50)$ & \\
\hline 1983 & Einstein MPC & $3.24 \pm 0.03$ & \\
\hline 1984 & EXOSAT LE & $4.3 \pm 0.7$ & \\
\hline 1984 & EXOSAT LE & $7.0 \pm 0.8$ & \\
\hline 1984 & EXOSAT ME & $0.75 \pm 0.25$ & \\
\hline 1990 & PSPC (Survey) & $0.536 \pm 0.046$ & \\
\hline 1991 & PSPC & $0.315 \pm 0.006$ & 0.12 \\
\hline 1993 & PSPC & 0.8 to 1.3 & 0.07 to 0.11 \\
\hline \multicolumn{4}{|l|}{ CP Pup } \\
\hline 1979 & Einstein IPC & $0.060 \pm 0.006(0.08)$ & \\
\hline 1990 & PSPC (Survey) & $0.061 \pm 0.021$ & \\
\hline 1993 & PSPC & $0.065 \pm 0.003$ & 0.07 \\
\hline \multicolumn{4}{|l|}{ GK Per } \\
\hline 1979-1980 & Einstein IPC & $0.178 \pm 0.016(0.33)$ & \\
\hline 1990 & PSPC (Survey) & $0.120 \pm 0.016$ & \\
\hline 1992 & PSPC & $0.213 \pm 0.004$ & 0.21 \\
\hline 1996 & ROSAT HRI & $0.065 \pm 0.003$ & \\
\hline \multicolumn{4}{|l|}{ V841 Oph } \\
\hline 1979 & Einstein IPC & $0.019 \pm 0.005(0.04)$ & \\
\hline 1990 & PSPC & $0.028 \pm 0.010$ & \\
\hline \multicolumn{4}{|l|}{ CP Lac } \\
\hline 1979 & Einstein IPC & $\leq 0.012(\leq 0.03)$ & \\
\hline 1993 & PSPC & $0.037 \pm 0.001$ & 0.65 \\
\hline \multicolumn{4}{|l|}{ T Sco } \\
\hline 1992 & PSPC & $0.0004 \pm 0.0001$ & \\
\hline \multicolumn{4}{|l|}{ DQ Her } \\
\hline 1979 & Einstein IPC & $\leq 0.030$ & \\
\hline 1992 & PSPC & $0.012 \pm 0.001$ & 0.56 \\
\hline \multicolumn{4}{|l|}{$\overline{\mathrm{RS} O p h}$} \\
\hline 1991 & PSPC & $0.012 \pm 0.002$ & \\
\hline 1992 & PSPC & $0.003 \pm 0.001$ & \\
\hline \multicolumn{4}{|l|}{ V446 Her } \\
\hline 1990 & PSPC (Survey) & $0.036 \pm 0.012$ & \\
\hline 1993 & PSPC & $\leq 0.013$ & \\
\hline
\end{tabular}

polars, has lower X-ray luminosity than the rest: are perhaps some IP-novae unusually under-luminous in Xrays?).

The All-Sky Survey detection of a source in the spatial error box of both V382 Sco and V720 Sco (Orio et al. 1992a) is ruled out examining the pointed observations 

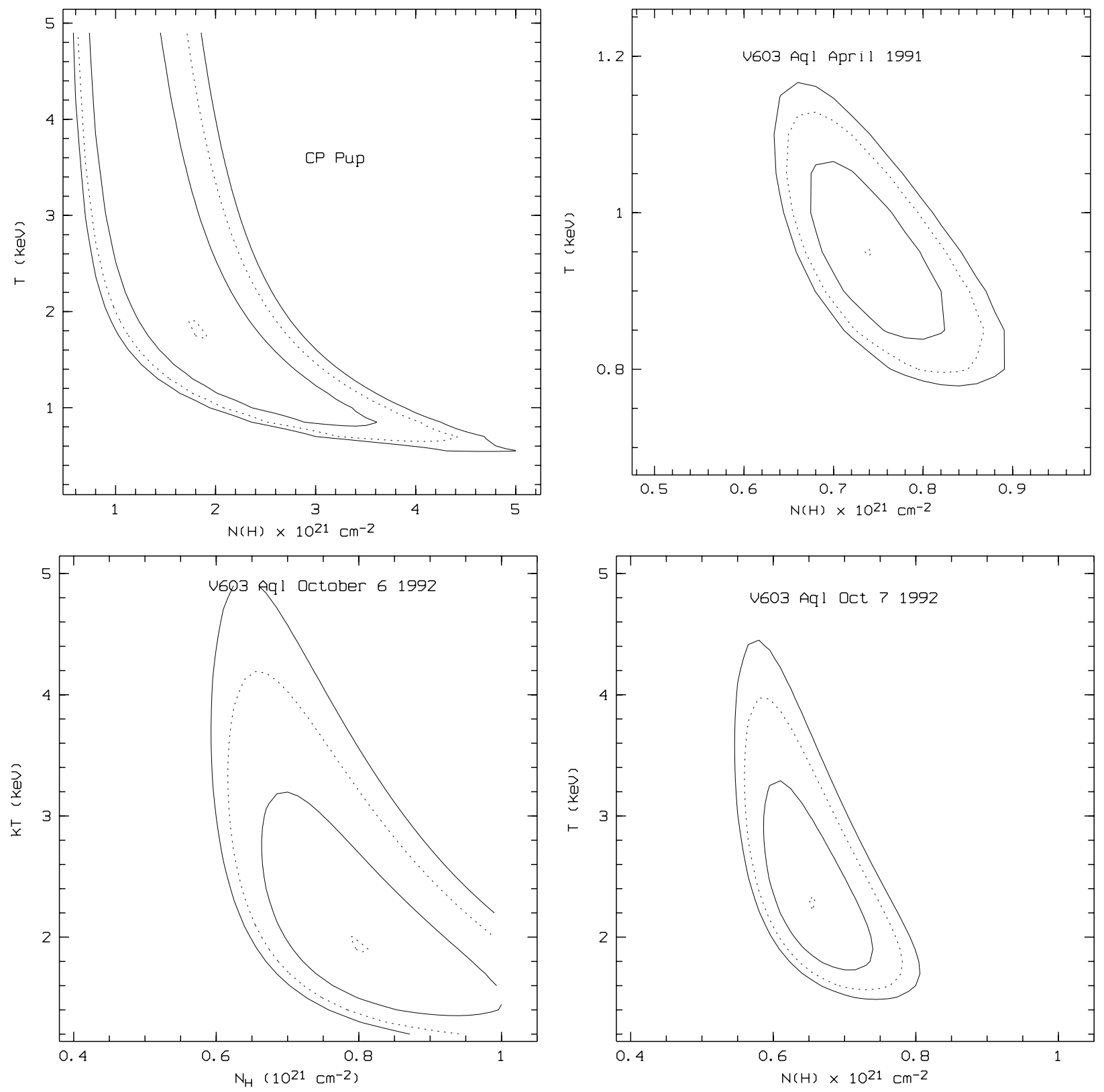

Fig. 2. 1, 2 and $3 \sigma$ confidence contours in the $N(\mathrm{H})$ versus $T$ (plasma temperature) plane for CP Pup and for V603 Aql in three different PSPC observations: in April 1991 and in two of the short observations taken in 1992, done on October 6 and 7 respectively (see Table 3). The small dotted contours indicate the best fit values. The best fit flux is $2.1 \times 10^{-12} \mathrm{erg}_{\mathrm{cm}}^{-2} \mathrm{~s}^{-1}$ for CP Pup, and for the three V603 Aql observations shown we obtained $7.9 \times 10^{-12} \mathrm{erg} \mathrm{cm}^{-2} \mathrm{~s}^{-1}, 10^{-11} \mathrm{erg} \mathrm{cm}^{-2} \mathrm{~s}^{-1}$, and $2.8 \times 10^{-11} \mathrm{erg} \mathrm{cm}^{-2} \mathrm{~s}^{-1}$ respectively.

(or the source is variable at quiescence). Only marginal detections were obtained for V446 Her and V841 Oph (observed only during the All-Sky Survey) and in the pointed observations of DQ Her, T Boo, and CP Lac.

For RR Pic the super-soft component is fitted with the a blackbody at $T_{\mathrm{BB}} \simeq 25 \mathrm{eV}$ and $F_{\text {bol }} \simeq 2 \times$ $10^{-13} \mathrm{erg} \mathrm{cm}^{-2} \mathrm{~s}^{-1}$, much too low to be originated on the WD surface. There is an additional, less luminous hard component. The parameters are not well constrained, although a Raymond-Smith model with $k T \simeq 1 \mathrm{keV}$ and flux $F_{\mathrm{x}} \simeq 5 \times 10^{-14} \mathrm{erg} \mathrm{cm}^{-2} \mathrm{~s}^{-1}$ fits the data reasonably. We favour the interpretation of van Teeseling et al.
(1996) that the system is a polar, despite the low X-ray luminosity. On the basis of the X-ray spectral characteristics, this is the only new suspect magnetic system in all our sample. For GK Per, already known instead to be an IP, a reasonable fit to the harder spectral component (the one attributed to accretion, and corresponding to $\simeq 75 \%$ of the total X-ray flux) gives $N(\mathrm{H})=2 \times 10^{22} \mathrm{~cm}^{-2}$, $k T=870 \mathrm{eV}$ and flux $6.5 \times 10^{-12} \mathrm{erg} \mathrm{cm}^{-2} \mathrm{~s}^{-1} . N(\mathrm{H})$ is thought to be partially due to the nebula.

We are puzzled by the different behaviour of the two X-ray brightest novae, V603 Aql and CP Pup. They displayed, however, similar optical characteristics 
Table 5. Assumed distances, derived X-ray luminosities, optical magnitude $V$, ratio of optical to X-ray luminosity, presence of long term (l.t.) and orbital (orb.) variability for accreting novae detected with ROSAT. For V841 Oph and V446 Her we assumed a distance of $1 \mathrm{kpc}$. " $\mathrm{n}$ " means that variability is ruled out in the ROSAT exposures. The magnitude in boldface for $\mathrm{T}$ Sco is in the $B$ band.

\begin{tabular}{lcccccc}
\hline Nova & $\begin{array}{c}d \\
(\mathrm{kpc})\end{array}$ & $\begin{array}{c}L_{\mathrm{x}} \times 10^{32} \\
\mathrm{erg} \mathrm{s}^{-1}\end{array}$ & $\begin{array}{c}L_{\mathrm{x}} / L_{\mathrm{opt}} \\
\times 10^{-2}\end{array}$ & l.t. & orb. \\
\hline V603 Aql & 0.43 & 2 & 11.7 & 1.4 & $\mathrm{x}$ & $\mathrm{n}$ \\
GK Per & 0.6 & 3.5 & 13.0 & 2.8 & $\mathrm{x}$ & \\
CP Pup & 0.7 & $0.59-5.9$ & 15.0 & 4.8 & & $?$ \\
V841 Oph & 1.0 & 0.3 & 13.4 & 1.6 & $?$ & \\
CP Lac & 1.15 & 3.8 & 16.6 & 1.1 & $?$ & \\
T Sco & 9.1 & 33 & 22.2 & 4.8 & & \\
DQ Her & 0.33 & 0.03 & 14.5 & 0.005 & & \\
RS Oph & 1.2 & $0.28-1.64$ & 12.5 & 0.2 & $\mathrm{x}$ & \\
RR Pic & 0.26 & 0.02 & 12.2 & 0.06 & $\mathrm{x}$ & $\mathrm{n}$ \\
V446 Her & 1 & 0.72 & 18.0 & 3.3 & $\mathrm{x}$ & \\
QU Vul & 2.6 & 0.18 & 19.0 & 1.7 & & \\
\hline
\end{tabular}

in outburst (Payne-Gaposhkin 1957) and at quiescence (the super-hump phenomenon). We rule out significant changes in the X-ray luminosity and spectrum of CP Pup from Einstein times. The opposite is true for V603 Aql. In the early $80^{\prime}$ s the count rate measured with Einstein varied by more than a factor of 3 within a couple of years (see Table 4). Drechsel et al. (1987) estimated $L_{\mathrm{x}} \simeq$ $3 \times 10^{33} \mathrm{erg} \mathrm{s}^{-1}$ and plasma temperature $k T=20-30 \mathrm{keV}$. There must be, however, even three components in the Xray flux of this nova, because Mukai (2000) showed that the ASCA spectrum can be fitted with two components at 1 and $9 \mathrm{keV}$. ROSAT is sensitive only to the lowest energy component, which instead must have been missing for CP Pup. In Fig. 2 we show the spectral probability contours in the $N(\mathrm{H})$ vs. $k T$ plane for the only pointed observation of CP Pup, and for three representative different observations of V603 Aql. From 1991 to 1992 the PSPC count rate measured for V603 Aql increased by a factor of 3, and during the 1992 observations it varied by a factor of $60 \%$ within 5 days. The spectrum in the PSPC range hardened in 1992 but did not seem to vary very much over hours and days (see Fig. 2). Drechsel et al. (1987) found irregular small scale variations over-imposed on the long scale one, and eclipses along the orbital period. In the light curve derived from 27 successive observations done in 1992 for approximately $1000 \mathrm{~s}$ each and spaced few hours apart (Fig. 3), no variations with the orbital periods (spectrometric and photometric) in the ROSAT range are present at a $3 \sigma$ confidence level. There is still flickering on short $(\simeq 10-100 \mathrm{~s})$ time scales. The ROSATPSPC light curve over 120 hours of observations in 1992 (shown in Fig. 3) gives a very interesting indication of a $\simeq 60$ hour periodicity, which needs to be verified but is observed at optical wavelengths and corresponds to the

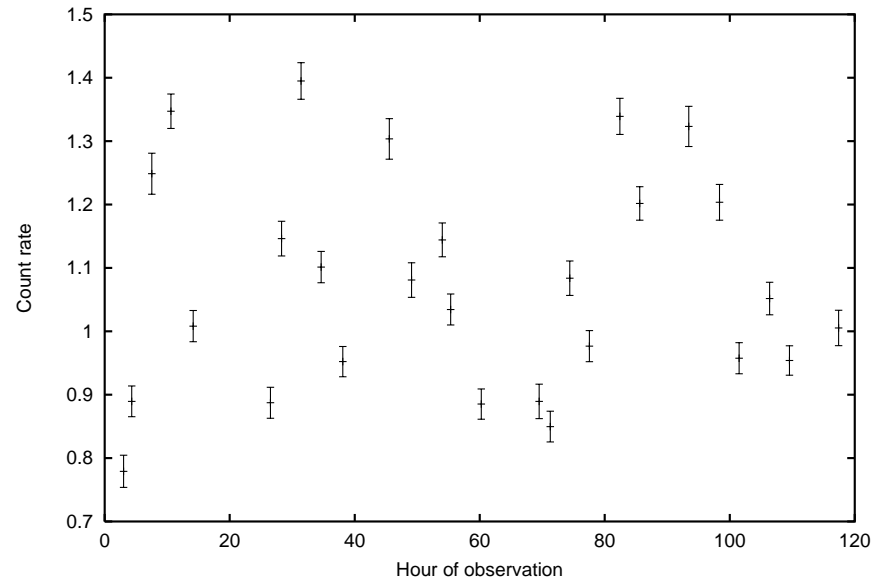

Fig. 3. The ROSAT PSPC light curve of V603 Aql observed intermittently for almost 120 hours in October 1992. Each point is the average of an observation lasting for about $1000 \mathrm{~s}$.

beat period (Udalski \& Schwarzenberg-Czerny 1989). For CP Pup (see also Balman et al. 1995), as the open contours seem to imply, there is no soft component and most likely the plasma temperature is above the ROSAT range. $\mathrm{X}$-ray modulations with the orbital period (Balman et al. 1995) and the high X-ray luminosity seem to be the signatures of an IP, however this interpretation is ruled out by Patterson \& Warner (1998).

Altogether, not only V603 Aql but 5 out of 11 objects seem to be variable X-ray sources: first of all RS Oph, which was almost three times less luminous in an unpublished serendipitous observation in 1991 than when observed by us in 1992 (Orio 1993). GK Per, V841 Oph and RR Pic also show hints of long term variability. The IPC-PSPC count rate conversion is done with PIMMS assuming $k T=1 \mathrm{keV}$.

The most important conclusion to be drawn from the ROSAT database is that there is no significant difference in the X-ray luminosity of dwarf novae and novae. Otherwise, the selection effect due to the distance would be compensated by the larger X-ray luminosity even at quiescence. As Table 5 shows there is also no clear correlation between X-ray luminosity $L_{\mathrm{x}}$ and the visual magnitude $V$ at minimum. The ratio $L_{\mathrm{x}} / L_{\mathrm{opt}}$, where $L_{\mathrm{x}}$ is the $\mathrm{X}$-ray luminosity in the $0.2-2.4 \mathrm{keV}$ range, varies greatly in the detected object and is low: $L_{\mathrm{x}} / L_{\mathrm{opt}}<0.03$ for most detected novae, and $L_{\mathrm{x}} / L_{\mathrm{opt}}<0.01$ for the non-detected objects. For those CV which, like most novae show strong emission lines at quiescence, and are likely to have optically thin disks, Patterson \& Raymond 1985) find typically $L_{\mathrm{x}} / L_{\mathrm{opt}} \simeq 0.03$. This ratio is only $\leq 0.01$ in erupting dwarf novae, where most of the boundary layer emission is thought to be radiated in the EUV range (Mauche et al. 1998). Is this also true for classical novae?

At least, as we show in Table 6, we rule out that the mass transfer rates inferred from the X-ray luminosity in the ROSAT range are representative. The accretion rates $\dot{M}_{\mathrm{x}}$ in the table were calculated assuming a variation of 
Table 6. Comparison of mass accretion rate approximately derived with different methods (in $M_{\odot} \times 10^{-10} \mathrm{yr}^{-1}$ ). References are in the text. The second column indicates number of years elapsed between the outburst and the X-ray observation.

\begin{tabular}{lccccl}
\hline Nova & years & $\dot{M}_{\mathrm{x}}(10)$ & $\dot{M}_{\mathrm{IR}}(10)$ & $\dot{M}_{\mathrm{opt}}(10)$ & $\dot{M}_{\mathrm{UV}}(10)$ \\
\hline V603 Aql & $73-74$ & 1 & 1 & 10 & 100 \\
GK Per & 92 & 1.6 & & 100 & \\
CP Pup & 50 & $0.35-3.5$ & & 0.3 & \\
V841 Oph & 152 & 1.8 & 30 & 1.7 & \\
CP Lac & 57 & 2.3 & 4000 & & \\
T Sco & 132 & 20 & & & \\
DQ Her & 58 & 0.02 & 6 & 300 & 50 \\
RS Oph & 7 & $0.1-1$ & & & \\
RR Pic & 68 & 0.01 & 1 & & \\
V446 Her & $32-33$ & 0.04 & 4 & & \\
PW Vul & $7-9$ & $<6$ & 34 & & \\
V1500 Cyg & $26-27$ & $<0.04$ & & 0.03 & 0.035 \\
QU Vul & 5 & 0.01 & & & \\
\hline
\end{tabular}

the analytical formula of Patterson \& Raymond (1985) adapted to the ROSAT range:

$\dot{M}_{\mathrm{x}}=\frac{L_{\mathrm{x}}(0.2-2.4 \mathrm{keV})}{11.5 \times 10^{31} M_{0.7}^{1.8}} \mathrm{erg} \mathrm{s}^{-1}$

where $M_{0.7}=M_{\mathrm{WD}} / 0.7 M_{\odot}$. In this formula a RaymondSmith model of thermal plasma with $k T=2 \mathrm{keV}$ and $N(\mathrm{H})=10^{21} \mathrm{~cm}^{-2}$ is assumed, and the fraction of gravitational energy which is re-radiated by the boundary layer is 0.5 . The formula works for other $\mathrm{CV}$ for $\dot{M} \leq 10^{-16} \mathrm{~g} \mathrm{~s}^{-1}$. Assuming for the sake of simplicity always $M_{\mathrm{WD}}=1.3 M_{\odot}$, we derived the approximate mass transfer rates $\dot{M}_{\mathrm{x}}$ in Table $6 . \dot{M}$ inferred from infrared observations (Weight et al. 1994), from the optical magnitude and its the rate of decline (Dürbeck 1992), from the optical magnitude, and from UV observations (Patterson 1984 and references therein) often differs from $\dot{M}_{\mathrm{x}}$ by much more than one order of magnitude (which would still be justified by a lower WD mass or lower fraction of reradiated energy). Whether this "missing boundary layer emission" is due to the accretion energy being re-radiated mostly below $0.2 \mathrm{keV}$, is a possibility that we cannot rule out.

There is no correlation of the derived $\dot{M}_{\mathrm{x}}$ with the post-outburst age as predicted by the hibernation theory (Shara et al. 1986). According to this theory, accretion occurs at a very high rate in the last 50-100 years before the outburst and for at least $\simeq 10$ years after. Concerning the time before the outburst, serendipitous observations were done with the ROSAT PSPC in the last 1 to 4 years before the outburst for only three Galactic novae, V1419 Aql, BY Cir and CP Cru. The $3 \sigma$ flux upper limits are of the order of $10^{-13} \mathrm{erg}^{-2} \mathrm{~cm}^{-1}$. We derive $L_{\mathrm{x}} \leq 2 \times 10^{30} \mathrm{erg} \mathrm{s}^{-1}$ for CP Cru and BY Cir (arbitrarily assuming a $d=1 \mathrm{kpc}$ ), and $L_{\mathrm{x}} \simeq 10^{31} \mathrm{erg} \mathrm{s}^{-1}$ for $\mathrm{N} \mathrm{Aql}$ 1993 (assuming $d=2.7 \mathrm{Kpc}$, from Kamath et al. 1997).
Two of the novae were moderately fast and one was fast (according to the theory, the faster the nova in outburst the larger the accretion rate).

\section{Conclusions}

1. Only $<20 \%$ of classical and recurrent novae have been observed as super-soft X-ray sources at some snapshot in time. If turn-off of the central source occurs within a few months, the accreted envelope is depleted more efficiently than foreseen by the models. We suggest three possible causes to explain this fact: a) a line driven wind depleting hydrogen burning material at the end of the outburst (Starrfield 1997, private communication) b) a magnetically driven wind (e.g. Orio et al. 1992b), c) a wind due to the secondary in the ejected shell (i.e. "common envelope" type of phenomenon).

2. More than half of all classical and recurrent novae were observed as transient hard X-ray sources in the first two years after the outburst. The origin is attributed to shocked ejecta. The shell may cool within a couple of years but it may also emit X-rays for up to a century under particular conditions, like previously existing circumstellar material (GK Per) or a wind continuing after the outburst (RR Tel).

3. The average X-ray luminosity of quiescent novae in the ROSAT range is not higher than $L_{\mathrm{x}} \simeq$ few times $10^{30} \mathrm{erg} \mathrm{s}^{-1}$, measured on average for dwarf novae by Verbunt et al. (1997). Our conclusion is based mainly on upper limits, because the majority of the Galactic novae were not detected. We remark that the X-ray brightest classical novae detected in quiescence are all fast novae.

4. There is no clear correlation of X-ray and visual luminosity. For the majority of quiescent novae $L_{\mathrm{x}} / L_{\mathrm{opt}} \leq$ 0.01. The mass transfer rates derived from the X-ray luminosity are much lower than those inferred from other wavelength ranges, implying a "missing boundary layer". Whether this is due simply to the fact that the accretion energy is not re-radiated in X-rays but almost entirely in the EUV range, like for DN in outburst (Mauche 1998), at the moment cannot be proved or disproved.

5. There is no correlation between X-ray luminosity and time elapsed before or after the outburst.

6 . The nova that was best studied with the ROSAT PSPC at quiescence, V603 Aql (also the most luminous) has variable X-ray spectrum and luminosity on time scales of 1 year and of few days. The recurrent nova RS Oph and a few classical novae also seem to have been at different X-ray luminosity (or spectral energy distribution) during the ROSAT lifetime. Most likely the luminosity level, or the spectral distribution, was also different in the observations done with Einstein approximately 10 years earlier. A self-consistent theory of accretion in classical novae has to take this X-ray variability into account. Monitoring the long term X-ray light curve of quiescent novae in an efficient way and explaining its characteristics is a challenge we have ahead. 
Acknowledgements. Both M. O. and H. Ö. acknowledge support of the Graduate School of the University of Wisconsin during the course of this project. The project was also partially supported by the Italian Space Agency ASI and by the NASA grant 98-03-ADP-042. We thank Jim MacDonald for the white dwarf atmospheric models.

\section{References}

Anupama, G. C., Dürbeck, H. W., Prabhu, T. P., \& Jain, S. K. 1992, A\&A, 263, 87

Anupama, G. C., \& Mikolajewska, J. 1999, A\&A, 344, 177

Balman, S., Krautter, J., \& Ögelman, H. 1998, ApJ, 499, 395

Balman, H., \& Ögelman, S. 1999, ApJ, 518, L111

Balman, S., Orio, M., \& Ögelman, H. 1995, ApJ, 449, L47

Becker, H., \& Marshall, F. E. 1981, ApJ, 244, L93

Benjamin, R. A., \& Dinerstein, H. L. 1990, AJ, 100, 1588

Brecher, K., Ingham, W. H., \& Morrison, P. 1977, ApJ, 213, 49

Burwitz, V., \& Reinsch, K. 2000, in 1999 Annual Scientific Meeting of the German Astr. Soc., AG Abstract Service, vol. 15

Chlebowsky, T., \& Kaluzny, J. 1988, Acta Astr., 38, 329

Contini, M., Orio, M., \& Prialnik, D. 1995, MNRAS, 275, 195

Cropper, M. 1990, Space Sci. Rev., 54, 1950

Della Valle, M., Gilmozzi, R., Bianchini, A., \& Esenoglu, H. 1997, A\&A, 325, 1151

Della Valle, M., \& Livio, M. 1995, ApJ, 452, 704

Diplas, A., \& Savage, B. D. 1994, ApJ, 427, 274

Drechsel, A., et al. 1987, A\&A, 126, 357

Dürbeck, H. W. 1988, ApL, 27, 286

Dürbeck, H. W. 1992, MNRAS, 258, 629

Fujimoto, M. Y. 1982, ApJ, 257, 752

Gehrz, R. D., et al. 1990, ApJ, 352, 307

Gonzalez-Riestra, R., Orio, M., \& Gallagher, J. 1998, A\&AS, 129,23

Greiner, J. 2000, New Astron., 353, 998

Greiner, J., Schwarz, R., Hasinger, J., \& Orio, M. 1996, A\&A, 312,88

Harrison, T. E., \& Gehrz, R. D. 1988, AJ, 96, 1001

Jordan, S., Mürset, U., \& Werner, K. 1994, A\&A, 283, 475

Kahabka, P., Hartmann, H. W., Parmar, A. N., \& Negueruela, T. 1999, A\&A, 347, 243

Kahabka, P., \& van den Heuvel, E. P. J. 1997, ARA\&A, 36, 69

Kamath, U. S., Anupama, G. C., Ashok, N. M., \& Chandrasekhar, T. 1997, AJ, 114, 2671

Kato, M. 1997, ApJS, 113, 121

Khruzina, T. S., \& Shugarov, S. Y. 1991, Atlas of Cataclysmic variables: U Geminorum Stars, ed. A. M. Cherepashchuk (Moscow: Moscow University press)

Krautter, J., et al. 1996, ApJ, 456, 788

Krautter, J., \& Williams, R. E. 1989, ApJ, 341, 968

Lepine, S., Shore, S. M., Livio, M., \& Zurek, D. 1999, ApJ, 522, L121

Lloyd, H. M., et al. 1992, Nature, 356, 222

Lloyd, H. M., \& O'Brien, T. J. 1994, Ap\&SS, 216, 161

Lloyd, H. M., O'Brien, T. J., \& Bode, M. F. 1997, MNRAS, 284,137

MacDonald, J., \& Vennes, S. 1991, ApJ, 373, L51

Mac Laughlin, D. B. 1960, in Stars and Stellar Systems, vol. 6, Stellar Atmospheres, ed. J. L. Greenstein (Chicago: Univ. Chicago Press), 585
Mason, K. O., Cordova, F. A., Bode, M. F., \& Barr, P. 1987, in RS Oph and the recurrent nova phenomenon, ed. M. F. Bode (London: VNU Science press), 167

Mathis, J. S., Cohen, D., Finley, J., \& Krautter, J. 1995, ApJ, 449,320

Mauche, C. 1998, ASP Conf. Ser., 137, 113

Mukai, K. 2000, New Astron. Rev., 44, 9

Mukai, K., \& Ishida, M. 2001, preprint [astro.ph 1026M]

O'Brien, T. J., \& Lloyd, H. M. 1994, Ap\&SS, 216, 167

O'Brien, T. J., Lloyd, H. M., \& Bode, M. F. 1994, MNRAS, 271,155

Ögelman, H., Beuermann, K., \& Krautter, K. 1984, ApJ, 287, 310

Ögelman, H., Krautter, J., \& Beuermann, K. 1987, A\&A, 177, 110

Ögelman, H., Orio, M., Krautter, J., \& Starrfield, S. 1993, Nature, 361, 331

Ögelman, H., \& Orio, M. 1995, in Cataclysmic Variables, ed. A. Bianchini, M. Della Valle, \& M. Orio (Kluwer), 11

Orio, M. 1993, A\&A, 274, L41

Orio, M., Balman, S., Della Valle, M., Gallagher, J., \& Ögelman, H. 1996, ApJ, 466, 410

Orio, M., \& Greiner, J. 1999, A\&A, 344, L13

Orio, M., \& Mukai, K. 2001, in preparation

Orio, M., et al. 1992a, Adv. Space Res., 13, 12

Orio, M., et al. 2001, MNRAS, in press

Orio, M., \& Ögelman, H. 1996, BAAS, 188, 28.03

Orio, M., \& Ögelman, H. 1998, in Wild Stars in the Old West: Proceedings of the 13th North American Conference on Cataclysmic Variables, ASP Conf. Ser., 137, 560

Orio, M. 1999, Phys. Rep., 311, 419

Orio, M., Torroni, V., \& Ricci, R. 1999a, IAU Circ. 7196

Orio, M., Trussoni, E., \& Ögelman, H. 1992b, A\&A, 257, 548

Patterson, J. 1984, ApJS, 54, 443

Patterson, J. 1994, PASP, 106, 209

Patterson, J., \& Raymond, J. C. 1985, ApJ, 292, 535

Patterson, J., \& Warner, B. 1998, PASP, 110, 1026

Payne-Gaposhkin, C. 1957, The Galactic Novae (Amsterdam: North Holland)

Prialnik, D. 1986, ApJ, 310, 222

Ramsay, G., Cropper, M., \& Mason, K. O. 1995, MNRAS, 276, 1382

Richman, H. R. 1996, ApJ, 462, 404

Saizar, P., Starrfield, S., \& Ferland, G. J. 1992, ApJ, 398, 651

Sekiguchi, K., et al. 1989, MNRAS, 236, 611

Schwarz, G. J., et al. 1997, MNRAS, 284, 669

Shanley, L., Ögelman, H. M., Gallagher, J., Orio, M., \& Krautter, J. 1995, ApJ, 438, L95

Udalski, A., \& Schwarzenberg-Czerny, A. 1989, Acta Astron., 39,125

van Teeseling, A., Beuermann, K., \& Verbunt, F. 1996, A\&A, 315,467

Verbunt, F., Bunk, W. H., Ritter, H., \& Pfeffermann, E. 1997, A\&A, 327, 602

Vrtilek, S. D., Silber, A., Raymond, J. C., \& Patterson, J. 1994, ApJ, 425, 787

Weight, A., Evans, A., Naylor, T., Wood, J. H., \& Bode, M. F. 1994, MNRAS, 26, 721

Williams, R. E. 1992, AJ, 204, 725

Williams, R. E. 1994, ApJ, 426, 279

Wu, C., \& Kester, D. 1977, A\&A, 58, 331

Zimmermann, H. V., et al. 1994, MPE Rep., 257 